\title{
Dietary cocoa protects against colitis-associated cancer by activating the Nrf2/Keap1 pathway
}

\begin{abstract}
Colorectal cancer (CRC) is the third most common malignancy in males and the second most common cancer worldwide. Chronic colonic inflammation is a known risk factor for CRC. Cocoa contains many polyphenolic compounds that have beneficial effects in humans. The objective of this study is to explore the antioxidant properties of cocoa in the mouse model of azoxymethane (AOM)/dextran sulfate sodium (DSS)-induced colitis-associated cancer, focusing on the activation of Nrf2 signaling. Mice were treated with AOM/DSS and randomized to receive either a control diet or a 5 and $10 \%$ cocoa diet during the study period. On day 62 of the experiment, the entire colon was processed for biochemical and histopathological examination and further evaluations. Increased levels of malondialdehyde (MDA) were observed in AOM/DSS-induced mice; however, subsequent administration of cocoa decreased the MDA. Enzymatic and nonenzymatic antioxidants, such as superoxide dismutase, catalase, glutathione peroxidase and glutathione reductase, were decreased in the AOM/DSS mice. Cocoa treatment increases the activities/levels of enzymatic and nonenzymatic antioxidants. Inflammatory mediators, such as inducible nitric oxide synthase (iNOS) and cyclooxygenase (COX)-2, were elevated during AOM/DSS-induction, and treatment with 5 and $10 \%$ cocoa effectively decreases the expression of iNOS and COX-2. The NF-E2-related factor 2 and its downstream targets, such as NQO1 and UDP-GT, were increased by cocoa treatment. The results of our study suggest that cocoa may merit further clinical investigation as a chemopreventive agent that helps prevent CAC.
\end{abstract}

Keyword: Colitis-associated cancer; Cocoa; Nrf2; iNOS; COX-2 